\title{
Comparación de las manifestaciones periodontales en una muestra de consumidores de chimú y cigarrillo en Villavicencio, Colombia
}

Diana Forero ${ }^{*}, \mathrm{MsC}_{\text {. DDs. }}$, Édgar Espinosa, DDs. ${ }_{1}$, Luis Alexys Pinzón-

Castro, Ph.D.

${ }_{1}$ Universidad Cooperativa de Colombia, Villavicencio, Colombia

Recibido: 29 de octubre del 2014. Aprobado: 3 de diciembre del 2014.

*Autor de correspondencia: Diana Forero Escobar. Facultad de Odontología, Universidad Cooperativa de Colombia, Villavicencio, Colombia. Kilómetro 2 Vía Acacías. Teléfono: (57) 6 6818850, ext. 8582.

Correo electrónico: diana.foreroe@ucc.edu.co

Cómo citar este artículo: Forero D, Espinosa E, Pinzón-Castro LA. Comparación de las manifestaciones periodontales en una muestra de consumidores de chimú y cigarrillo en Villavicencio, Colombia. Rev Nac Odontol. 2015;11(20):41-45.

doi: http://dx.doi.org/10.16925/od.v11i20.802

Resumen. Introducción: el tabaco de mascar, conocido en Colombia como chimú, tiene como principio activo la nicotina, alcaloide líquido, oleoso e incoloro que atraviesa la mucosa bucal, factor desencadenante y agravante de enfermedades periodontales. El propósito de este estudio fue determinar la diferencia en pacientes que consumen tabaco mascado (chimú), en comparación con un grupo de fumadores de cigarrillo y un grupo de no consumidores. Métodos: estudio de casos y controles anidado en un corte transversal, con muestra por conveniencia de 90 sujetos (18-40 años). Se hizo un examen intraoral que incluía periodontograma e índice de placa bacteriana O'Leary, para clasificar la enfermedad periodontal según la Academia Americana de Periodoncia y las retracciones gingivales según Miller. Resultados: en total, un 26,7\% de los sujetos que consumían chimú presentó enfermedad periodontal; 73,3\%, gingivitis; $63,3 \%$, retracciones gingivales, y $40 \%$, hiperplasia gingival. En pacientes fumadores, un 63,3\% presentó enfermedad periodontal; $36,7 \%$, gingivitis; $26,7 \%$, recesiones gingivales, y $20 \%$, hiperplasia gingival. En pacientes no consumidores de tabaco, se encontró que 3,3\% presentaba enfermedad periodontal; $96,7 \%$, gingivitis; $20 \%$, retracciones gingivales, y $16,6 \%$, hiperplasias gingivales. Asociación entre chimú y cigarrillo: enfermedad periodontal ( $\mathrm{P}=0,004$, OR 0,21 IC 95\% 0,70-0,631), gingivitis ( $\mathrm{P}=0,004$ oR 4,75 IC 95\% $1,58-14,24)$, retracciones gingivales ( $\mathrm{P}=0,004$, oR 4,75 IC $95 \% 1,58-14,24)$, hiperplasia gingival (valor $\mathrm{P}: 0,091$ ). Conclusiones: consumir chimú es menos nocivo que fumar, en cuanto a la aparición de enfermedad periodontal; sin embargo, es un factor de riesgo para la aparición de gingivitis y retracciones gingivales. La hiperplasia gingival no está asociada con los hábitos de fumar o mascar chimú.

Palabras clave: chimú, cigarrillo, enfermedad periodontal, hiperplasia, retracción gingival. 


\title{
Periodontal Manifestation Comparison in a Group of Chimu Consumers and Smokers in Villavicencio, Colombia
}

\begin{abstract}
Introduction: the active ingredient of chewing tobacco, known as chimú in Colombia, is nicotine, a liquid, oily and colorless alkaloid that goes through the oral mucosa, which is a triggering factor and further problem in periodontal diseases. The objective of this analysis was to determine the difference in patients consuming chewing tobacco (chimu), compared to a group of cigarette smokers and a group of non-smokers. Methods: a case and cross-sectional study, with a sample, for convenience purposes, of 90 subjects (18 to 40 years). An intra-oral examination was conducted, which included a periodontogram, as well as the O'Leary index, both to classify the periodontal disease according to the American Academy of Periodontology, and gingival retractions according to Miller. Results: a total of $26.7 \%$ of the subjects who chewed chimu had periodontal disease; $73.3 \%$ gingivitis, 63.3 gingival retractions; and $40 \%$ gingival hyperplasia. As for smokers, $63.3 \%$ had periodontal disease; $367 \%$, gingivitis; $26.7 \%$, gingival retractions, and $20 \%$, gingival hyperplasia. As for patients who did not consume tobacco, $3.3 \%$ had periodontal disease; $96.7 \%$, gingivitis; $20 \%$, gingival retractions, and $16.6 \%$, gingival hyperplasia. Association between chimu and cigarettes: periodontal disease ( $\mathrm{P}=0,004$, OR 0,21 CI 95\% 0,70-0,631), gingivitis ( $\mathrm{P}=0,004$ oR 4,75 CI 95\% 1,58-14,24), gingival retractions ( $\mathrm{P}=0,004$, OR 4,75 CI 95\% 1,58-14,24), gingival hyperplasia (valor $\mathrm{P}: 0,091)$. Conclusions: chimu consumption is less harmful than smoking, as regards periodontal disease. It is, however, a risk factor for gingivitis and gingival retraction. Gingival hyperplasia is not associated with the smoking and tobacco chewing habits.
\end{abstract}

Keywords: chimu, cigarette, periodontal disease, hyperplasia, gingival retraction.

\section{Comparação das manifestações periodontais em uma amostra de consumidores de chimu e cigarro na cidade de Villavicencio, na Colômbia}

\begin{abstract}
Resumo. Introdução: o tabaco mastigável, na Colômbia conhecido de "chimu", tem como princípio ativo a nicotina, alcaloide líquido, oleoso e incoloro que passa pela mucosa bucal, fator desencadeante e agravante de doenças periodontais. $\mathrm{O}$ escopo deste estudo foi determinar a diferença em pacientes que consumem tabaco mastigado (chimu), comparado com um grupo de fumadores de cigarro e um grupo de não consumidores. Métodos: estudo de casos e controles aninhado em um corte transversal, com amostra por conveniência de 90 sujeitos (18-40 anos). Foi feito um exame intrabucal que incluía PSR (Avaliação Periodontal Simplificada) e o índice de placa bacteriana O’Leary, para classificar a doença periodontal segundo a Academia Americana de Periodontia e as retrações gengivais segundo Miller. Resultados: em total, 26,7\% dos sujeitos que consumiam chimu apresentou doença periodontal; 73,3\%, gengivite; $63,3 \%$, retrações gengivais, e $40 \%$, hiperplasia gengival. Em pacientes fumadores, $63,3 \%$ apresentou doença periodontal; 36,7\%, gengivite; $26,7 \%$, recessões gengivais, e $20 \%$, hiperplasia gengival. Em pacientes não consumidores de tabaco, encontrou-se que 3,3\% apresentava doença periodontal; $96,7 \%$, gengivite; $20 \%$, retrações gengivais, e 16,6\%, hiperplasias gengivais. Associação entre chimu e cigarro: doença periodontal ( $\mathrm{P}=0,004$, OR 0,21 IC $95 \% 0,70-0,631)$, gengivite ( $\mathrm{P}=0,004$ OR 4,75 IC 95\% 1,58-14,24), retrações gengivais ( $\mathrm{P}=0,004$, OR 4,75 IC 95\% 1,58-14,24), hiperplasia gengival (valor P: 0,091). Conclusões: consumir chimu é menos nocivo do que fumar, no que diz respeito ao aparecimento de doença periodontal; no entanto, é um fator de risco para o aparecimento da gengivite e retrações gengivais. A hiperplasia gengival não está associada com os hábitos de fumar ou mastigar chimu.
\end{abstract}

Palavras-chave: chimu, cigarro, doença periodontal, hiperplasia, retração gengival. 


\section{Introducción}

El tabaco es una sustancia psicoactiva que viene en varias presentaciones, y la más común es el cigarrillo [1]. Fumar constituye uno de los factores de riesgo asociados con más frecuencia con el desarrollo de periodontopatías inflamatorias, e influye de un modo relevante en el curso y pronóstico de la enfermedad periodontal [2]. En la actualidad, aunque predomina el uso del tabaco fumado, las industrias tabacaleras están cambiando el mercado del cigarrillo por productos denominados "tabacos sin humo", basados en la premisa de que son inofensivos para la salud. Dentro de esta gama de productos, se encuentra el tabaco de mascar (en Colombia es conocido como chimú), que tiene como principio activo la nicotina, un alcaloide líquido, oleoso e incoloro que atraviesa la mucosa bucal y se absorbe lentamente; y cuyos niveles en la sangre son similares a los de un fumador de cigarrillo [3].

La forma de consumo del chimú es por vía oral; se coloca en la boca y luego se frota contra los dientes, la encía y la lengua, produciendo una fuerte salivación [3]. Es considerado un factor desencadenante y agravante de enfermedades periodontales como gingivitis, periodontitis, hiperplasias gingivales, pigmentaciones de la mucosa o alteraciones como leucoplasias, quetaratosis o eritoplasias [4].

En Colombia, los efectos nocivos del tabaco fumado (cigarrillo) en cavidad oral han sido ampliamente estudiados; sin embargo, no hay investigaciones que reporten los efectos del tabaco mascado (chimú). El propósito de este estudio es determinar si existe diferencia en la prevalencia de manifestaciones de enfermedades periodontales en pacientes que consumen tabaco mascado (chimú), comparados con un grupo de fumadores de cigarrillo y con un grupo de no consumidores de tabaco.

\section{Métodos}

Se llevó a cabo un estudio de casos y controles anidado en un corte transversal, con una muestra por conveniencia de 90 sujetos (18-40 años), dividida en tres grupos: 30 consumidores de chimú, 30 fumadores y 30 no consumidores de tabaco; se excluyeron sujetos con enfermedades sistémicas, en tratamiento farmacológico o con aparatología oral.

Se utilizó el índice de placa bacteriana O’Leary, y se clasificó la enfermedad periodontal según la Academia Americana de Periodoncia y las retracciones gingivales se tipificaron según Miller. Se hizo previa calibración de los examinadores en relación con un especialista en periodoncia (Kappa 0,87). El proyecto tuvo aval ético institucional según las normas que rigen la investigación en Colombia.

Después de la identificación de los pacientes, se hicieron controles de placa bacteriana y se utilizaron contrastes paramétricos, como análisis Anova, y no paramétricos, como la prueba de Kruscal-Wallis o la prueba u de Mann-Whitney. Esto dependió de la normalidad de los datos (con valores de P significativos menores que 0,05 ), los cuales visualizaron que los promedios de edad y de IPB se encontraban en el mismo subconjunto homogéneo de promedios al 95\% de confianza, de manera que los grupos fueran comparables.

Se hizo examen intraoral que incluía periodontograma, para el diagnóstico de enfermedad periodontal. Se hizo un análisis de probabilidad, en el que se identificó la prevalencia en cada uno de los grupos frente al tiempo y la frecuencia de consumo, utilizando tablas de contingencia como recurso de organización. A partir de las prevalencias más altas, se tomaron los casos y controles anidados. Los casos los constituyeron los pacientes que consumían chimú o cigarrillo y que presentaban la enfermedad, y los controles fueron aquellos que no la presentaban. Para medir el grado de asociación entre variables, se hizo la prueba de or (análisis de riesgo), con intervalos de confianza al $95 \%$.

\section{Resultados}

Se encontró que la edad media de los sujetos participantes en el estudio fue de 26,89 años, con una desviación estándar de 5,3 años; además, que ocho $(26,7 \%)$ de los sujetos que consumían chimú presentaron enfermedad periodontal; tres (10\%), enfermedad periodontal incipiente, y cinco $(16,7 \%)$, enfermedad periodontal moderada. Veintidós $(73,3 \%)$ sujetos consumidores de chimú presentaron gingivitis con pérdida de inserción preexistente, $19(63,3 \%)$ presentaron retracciones gingivales y 12 (40\%) presentaron hiperplasia gingival. Entre los pacientes que fumaban, se encontraron $19(63,3 \%)$ con enfermedad periodontal: 14 (46,7\%) tenían grado incipiente y cinco (16,7\%), grado moderado; $11(36,7 \%)$ presentaron gingivitis, ocho $(26,7 \%)$ tenían recesiones gingivales y seis (20\%) presentaron hiperplasia gingival (tabla 1). 
Tabla 1. Prevalencias de las enfermedades periodontales

\begin{tabular}{|l|c|c|c|c|c|c|}
\hline \multicolumn{1}{|c|}{ Grupo } & $\begin{array}{c}\text { Enfermedad } \\
\text { periodontal }\end{array}$ & Incipiente & Moderada & $\begin{array}{c}\text { Gingivitis con } \\
\text { pérdida } \\
\text { preexistente }\end{array}$ & Retracciones & Hiperplasia \\
\hline Chimú & $26,7 \%$ & $10 \%$ & $16,7 \%$ & $73,3 \%$ & $63,3 \%$ & $40 \%$ \\
\hline Cigarrillo & $63,3 \%$ & $46,7 \%$ & $16,7 \%$ & $36,7 \%$ & $26,7 \%$ & $20 \%$ \\
\hline
\end{tabular}

Fuente: elaboración propia

Tras determinar las prevalencias de las enfermedades, se procedió a hacer el estudio de casos y controles anidado en estos resultados, mediante pruebas ORatio con un intervalo de confianza al 95\%. Se encontró que la enfermedad periodontal estaba relacionada con el consumo de tabaco mascado y fumado; sin embargo, consumir chimú es menos nocivo que fumar para la aparición de esta enfermedad ( $P=0,004$, OR 0,21 IC $95 \% 0,07-0,631$ ). A su vez, el consumo de chimú es un factor de riesgo 4,7 veces mayor que el consumo de cigarrillo para la aparición de gingivitis $(\mathrm{P}=0,004$ OR 4,75 IC $95 \%$ 1,58-14,25); la posibilidad de padecer retracciones gingivales es 4,8 veces mayor en pacientes que consumen chimú que en aquellos que fuman ( $P=0,003$ or 4,8 IC 95\% 1,6-14,3), y no existe una asociación estadística entre la aparición de hiperplasia gingival y los hábitos de fumar o de mascar chimú (valor $\mathrm{P}=0,091$ ).

Al hacer el análisis de los datos con respecto al consumo de alcohol, se encontró que cuando los sujetos consumían alcohol con una frecuencia mayor a diez copas, la aparición de enfermedades era mayor. Sin embargo, al realizar las pruebas de chi cuadrado con una confiabilidad del $95 \%$, en ninguna de las patologías se encontró una posible asociación entre el consumo de tabaco y la frecuencia de bebidas alcohólicas.

\section{Discusión}

Los pacientes que consumían cigarrillo o chimú fueron menos propensos a la aparición de enfermedad periodontal, lo cual puede estar asociado a los cambios en la vasculatura gingival, en la homeostasis y en la respuesta inflamatoria e inmune que genera el humo y algunos componentes del tabaco [5], dado que los niveles de placa bacteriana, considerados la principal causa de gingivitis [6], fueron validados dentro de un mismo rango para este estudio.
En los sujetos que consumían chimú, se encontró un $70 \%$ de prevalencia de gingivitis, lo cual puede estar asociado con el tiempo que permanece la masa de chimú en la cavidad oral, pues el movimiento constante del chimú genera respuesta inflamatoria por trauma. El estudio de Manuel Sossa prueba que el chimú no inhibe la formación de la biopelícula dental que está presente en la enfermedad periodontal [3].

En cuanto a la aparición de periodontitis, el tabaco mascado (chimú) y el fumado (cigarrillo) se comportaron como factores predisponentes. Pero, en este estudio, los fumadores presentaron mayor prevalencia de la enfermedad periodontal que los consumidores de chimú. En relación con esto, D’Agostinia et al. consideran que el tabaco, en sus diferentes presentaciones, tiene relación con la progresión de la enfermedad periodontal [7], ya que su patogénesis está vinculada con una compleja interacción entre el medio ambiente microbiano, la genética y otros factores del huésped, lo que convierte el tabaco en el factor de riesgo ambiental más importante [7].

Swaminathan y Saud mencionan que fumar aumenta el nivel de radicales libres en los tejidos periodontales, que pueden ser responsables de la destrucción de los tejidos en la enfermedad periodontal [8]. Fisher y Taylor mencionan que los adultos que consumen tabaco sin humo son dos veces más propensos a tener enfermedad periodontal activa grave en cualquier sitio. Estos resultados indican que el tabaco sin humo, tanto como el cigarrillo, puede ser un factor de riesgo relevante para la enfermedad periodontal activa grave $[8,9]$.

Los resultados muestran cómo el consumo de tabaco fumado (cigarrillo) y mascado (chimú) son factores predisponentes en la aparición de enfermedades en la cavidad oral; contrario a las creencias populares y a los comerciantes del chimú, el tabaco mascado no es inofensivo para la salud oral.

Con respecto al tiempo de consumo de cigarrillo, la periodontitis fue la única patología que se 
asoció con mayor tiempo de consumo, mientras que la gingivitis disminuyó a medida que aumentaba el tiempo de consumo de cigarrillo. Por su parte, los pacientes que tenían menos de dos años de consumo de chimú presentaron gingivitis en su totalidad. Esto sugiere que el organismo, a medida que aumenta el tiempo de consumo de tabaco mascado, se adapta para disminuir la inflamación localizada [10]. Aquí se coincide con el estudio desarrollado en ratas Wistar, que concluyó que los cambios histológicos observados están directamente relacionados con el tiempo de exposición al chimú [3].

En este estudio, la aparición de las patologías no presentó resultados estadísticos que asocien el tiempo del consumo del chimú con periodontitis, hiperplasias o recesiones gingivales, lo cual reafirma que el chimú tiene un daño local por altas concentraciones y tiempo de estar en boca, que no ejerce concentración progresiva por el tiempo del consumo.

\section{Conclusiones}

La prevalencia de enfermedades periodontales como gingivitis, retracciones y recesiones gingivales es mayor en pacientes que consumen chimú que en pacientes que consumen cigarrillo.

La periodontitis es más prevalente en pacientes que fuman que en pacientes que mascan chimú o no consumen ningún tipo de tabaco.

Fumar es un factor de riesgo para desarrollar periodontitis, en comparación con el hábito de mascar chimú; sin embargo, el chimú es un factor de riesgo para desarrollar gingivitis y retracciones.

La aparición de hiperplasia gingival no está relacionada con el consumo de chimú o cigarrillo.

\section{Referencias}

[1] Organización Mundial de la Salud. Informe oms sobre la epidemia mundial de tabaquismo, 2011. Ginebra: OMs; 2011.

[2] Traviesas E, Rodríguez R. Tabaquismo, higiene bucal y periodontopatías inmunoinflamatorias crónicas en adultos del municipio Guanajay. Rev Cubana Estomatol. 2007;4(1)

[3] Sosa M, Urdaneta L, Jarpa P. Caracterización preliminar de la flora bacteriana en la biopelícula dental de individuos consumidores de Chimú. Rev Odont Los Andes. 2008;3(2):4-12.

[4] Jarpa P. Potencial mutagénico del tabaco de mascar venezolano. Rev Fac Farm. 2003;45(2):2-6.

[5] Matesanz P, Matos R, Bascones A. Enfermedades gingivales: una revisión de la literatura. Av Periodon Implantol. 2008;20(1):11-25.

[6] Nishida N, Yamamoto Y, Tanaka M, Kataoka K, Kuboniwa M, Nakayama K. Association between Involuntary Smoking and Salivary Markers Related to Periodontitis: A 2-Year Longitudinal Study. J Periodontol. 2008;79(12):2233-40.

[7] D’Agostinia F, Calcagno E, Micale T, La Maestra S, De Flora S, Cingano L. Cytogenetic Analysis of Gingival Epithelial Cells, as Related to Smoking Habits and Occurrence of Periodontal Disease. Int J Hyg Environ Health. 2013;216(1):71-5.

[8] Swaminathan M, Saud K. Estimation of Lipid Peroxides and Antioxidants in Smokers and Non-Smokers with Periodontitis. King Saud Univ J Dent Sci. 2013;4(2):53-6.

[9] Fisher MA, Taylor GW, Tilashalski KR. Smokeless Tobacco and Severe Active Periodontal Disease, NHANES III. J Dent Res. 2005;84(8):705-10.

[10] Andersson G, Axell T. Clinical Appearance of Lesions Associated with the Use of Loose and Portion-Bag Packed Swedish Moist Snuff: A Comparative Study. J Oral Pathol Med. 1989;18(1):2-7.

\section{Corrección}

Por un error involuntario, en este artículo no se incluyó al autor Luis Alexys Pinzón Castro. Se hace la corrección el 27 de octubre de 2015, en la versión digital. 